\title{
Philosophiques
}

\section{Symbole : fondements anthropobiologiques de la doctrine aristotélicienne du langage}

\section{Pierre Bellemare}

Volume 9, numéro 2, octobre 1982

URI : https://id.erudit.org/iderudit/203195ar

DOI : https://doi.org/10.7202/203195ar

Aller au sommaire du numéro

Éditeur(s)

Société de philosophie du Québec

ISSN

0316-2923 (imprimé)

1492-1391 (numérique)

Découvrir la revue

Citer cet article

Bellemare, P. (1982). Symbole : fondements anthropobiologiques de la doctrine aristotélicienne du langage. Philosophiques, 9(2), 265-279.

https://doi.org/10.7202/203195ar
Résumé de l'article

En De Interpretatione 16a 4-6, Aristote écrit que « ce qui est dans la voix est symbole des affections qui sont dans l'âme. " Ce terme de "symbole " n'est pas aussi clair qu'il le paraît d'abord, ainsi que le montre une analyse de l'usage qu'Aristote en fait dans ce passage et dans d'autres textes (De Int. 16a 28; Réfutations Sophistiques 1, 165 a 8; De Sensu 1, 437 a 15). En confrontant ces textes et en tenant compte de l'histoire du mot « $\sigma u ́ \mu \beta o \lambda o v$ » dans le grec antérieur et contemporain d'Aristote, il est possible de reconstituer les éléments d'une doctrine aristotélicienne du langage et de lier cette doctrine d'Aristote à ses préoccupations de biologiste et à sa compréhension de la structure du temps. 


\title{
SYMBOLE: FONDEMENTS ANTHROPOBIOLOGIQUES DE LA DOCTRINE ARISTOTELICIENNE DU LANGAGE*
}

\author{
par Pierre Bellemare
}

\begin{abstract}
RÉSUMÉ. En De Interpretatione 16a 4-6, Aristote écrit que «ce qui est dans la voix est symbole des affections qui sont dans l'âme.» Ce terme de "symbole» n'est pas aussi clair qu'il le paraît d'abord, ainsi que le montre une analyse de l'usage qu'Aristote en fait dans ce passage et dans d'autres textes (De Int. 16a 28; Réfutations Sophistiques 1, 165 a 8; De Sensu 1, 437 a 15). En confrontant ces textes et en tenant compte de l'histoire du mot " $\sigma u \mu \beta \circ \lambda o \nu$ " dans le grec antérieur et contemporain d'Aristote, il est possible de reconstituer les éléments d'une doctrine aristotélicienne du langage et de lier cette doctrine d'Aristote à ses préoccupations de biologiste et à sa compréhension de la structure du temps.
\end{abstract}

ABSTRACT. In De Interpretatione 16 a 4-6, Aristotle writes that "what is in the voice is a symbol of the affections that are in the soul." This term "symbol" is not as clear as it seems at first glance, as can be shown in an analysis of the way Aristotle uses it in this passage and elsewhere (De Int. 16 a 28; De Sophisticis Elenchis 1, 165 a 8; De Sensu 1, 437 a 15). Comparing these texts and taking into account the history of the word " oú $\beta$ o $\lambda o v$ " in Greek before Aristotle and in his own time, it is possible to reconstruct the elements of an aristotelian doctrine of language and to relate this doctrine to his studies in biology and to his theory concerning the structure of time.

La Nature est un temple où de vivants piliers laissent parfois sortir de confuses paroles; l'homme y passe à travers des forêts de symboles qui l'observent avec des regards familiers.

Beaudelaire, Les Fleurs du Mal

\footnotetext{
${ }^{*}$ Cet article, qui reprend les thèses de mon mémoire de maîtrise, a bénéficié des commentaires de MM. Jacques Poulain et Vianney Décarie, mes directeurs, et de M. John Thorp, qui en a commenté une première version au Congrès de l'ACP (1981).
} 
La plus tenace de nos idées reçues sur le langage, l'idée qu'il est d'abord le moyen que nous avons de nous communiquer mutuellement nos pensées, nous vient d'Aristote. En tout cas, cette conception est celle qui semble se dégager des premiers chapitres du traité De Interpretatione, où il est question du discours et de ses parties, et en particulier du tout premier chapitre, qui traite du langage d'un point de vue très général.

Cette idée est sûrement l'un des présupposés de ce texte, mais je conteste que ce soit là l'essentiel de ce qu'il a à nous dire. Je prétends même que toute une dimension capitale de ces pages a généralement échappé à leurs commentateurs. Dans cet article, je tâcherai de montrer qu'Aristote y envisage l'étude du langage dans une perspective "biologique" et même qu'on y trouve des signes qu'il subordonnait la question de l'essence du langage à celle de la nature de l'homme comme être vivant. Ces indices, jusqu'ici ignorés, je les trouve dans le vocabulaire qu'il y utilise.

Dans ce premier chapitre donc, Aristote donne une sorte de définition de la parole" ${ }^{1}$. "Ce qui est dans la voix, dit-il, est symbole (sumbola) de ce que ressent l'âme («les pathèmata qui sont dans l'âme») et ce qui est écrit (symbole) de ce qui est dans la voix» ${ }^{2}$. On notera tout de suite qu'Aristote y aborde le langage non d'un point de vue formel, comme un objet décanté, mais qu'il le prend encore tout habillé de matière sonore, si l'on peut dire, car il en parle comme de «ce qui est dans la phônè». Or, la "phônè» n'est pas pour lui une simple vibration de l'air, elle est du son incarné, elle est "en-animée» ( $\left.\mu_{\mu} \psi v \times \eta\right)^{3}$, comme il le précise au chapitre qu'il lui consacre dans le De Anima - son traité d'introduction à la collection de ses oeuvres de biologie. Dès cette première caractérisation de son objet d'étude, il l'inscrit donc dans cet horizon où je suggère qu'il faut replacer l'ensemble de ces chapitres introductifs.

Je ferai toutefois reposer le poids de ma thèse sur un autre mot de la même phrase, un mot qui y occupe une fonction aúrement plus importante. L'interprétation exacte de cette phrase

1. De la parole, dis-je, car le discours (logos) considéré comme un tout comportant des parties (d'un point de vue «structurel» donc) fera l'objet d'un chapitre particulier (De Int., 4).

2. De Int., 1, 16 à 4-6: «Ě̃

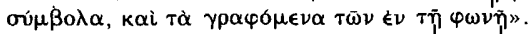

3. De Anima, II, $8420 \mathrm{~b} 6-421 \mathrm{a} 6$. 
essentielle dépend évidemment du sens qu'il faut donner au mot «symbole» qu'on y trouve. Ce terme, les commentateurs et les traducteurs d'Aristote qui se sont succédé depuis l'Antiquité l'ont envisagé de plusieurs façons différentes qui sont autant d'interprétations, explicites ou seulement implicites, de ce texte classique de l'histoire de la sémantique. Je commencerai par montrer que, bien qu'il nous semble familier, aucun des sens qu'on a tenté d'accorder à ce terme n'a réussi à en épuiser la signification. Puis, pour préparer la voie à ma propre interprétation, je décrirai, dans un détour philologique dont je prie le lécteur de bien vouloir excuser la longueur, le réseau de sens dans lequel était enchâssé "sumbolon» à l'époque d'Aristote. Enfin, à partir de ces renseignements et d'autres indications fournies par les textes, je proposerai ma lecture qui permettra d'élargir la problématique en direction de la biologie et des doctrines concernant les premiers principes de la nature, en particulier le temps.

La présence, dans l'espace d'un même court chapitre, d'une affirmation selon laquelle ce qui est dans la "phônè» est symbole des «pathèmata» qui sont dans l'âme et d'une autre ${ }^{4}$ qui veut que les "phônai» soient les signes (sèméia) de cès mêmes «pathèmata» a naturellement fait porter l'analyse du symbole sur ses rapports avec le signe. En voulant faire coïncider ces deux formules, certains interprètes ${ }^{5}$ ont nié ou minimisé la différence de ces concepts et plusieurs traducteurs étaient si fermement persuadés qu'elle n'existait même pas du tout qu'ils ont omis de reprendre dans leurs versions la distinction qu'ils trouvaient dans le grec. En tête de la liste de ceux qui ont ainsi «enlevé à leur lecteur la possibilité même de se poser une question sur cette nuance lexicale» ${ }^{6}$ figure Boëce ${ }^{7}$ qui rend les deux mots par un seul, «nota». Beaucoup de ceux qui l'ont suivi n'ont pas plus respecté

4. De Int., 1, 16 a 7.

5. En particulier, H. Steinthal (Geschichte der Sprachwissenschaft bei den Griechen und Römern, $2^{\mathrm{e}}$ éd., Berlin, Ferd. Dümmlers, 1890, vol. I, p. 186, la note).

6. Selon l'expression de N. Kretzman dans son article "Aristotle on spoken sound significant 'by convention" p. 7 in Ancient Logic and its modern interpretations (Reidel, Dordrecht/Boston, 1974).

7. Boëce, Commentarii in librum Aristotelis Peri Hermeneias, éd. Meiser, Teubner, Leipzig, 1878-1880, vol. I, p. 3: «sunt ergo ea quae sunt in anima passionum notae et ea quae scribuntur eorum quae sunt in voce» (1, 5-7) et plus loin (1, 9-11): "quorum autem haec primorum notae, eaedem omnibus passiones animae et quorum hae similitudines, res etiam eaedem.» 
la lettre de l'original: l'assimilation du «sumbolon» au «sèméion» est assez fréquente ${ }^{8}$ et l'inverse, quoique plus rare, n'est pas non plus sans exemple ${ }^{9}$. Toutefois, on trouve quelques excellentes versions ${ }^{10}$ qui respectent, chacune dans sa langue, cette différence lexicale qui est d'abord, je pense, l'expression d'une importante différence sémantique.

De la même façon, il y a des interprètes, Waitz ${ }^{11}$ et Aubenque $^{12}$, entre autres, qui ne regardent pas ce «symbole» comme un simple synonyme de «signe». Ils s'appuient sur le second chapitre du De Interpretatione qui traite du nom. Aristote, certes, y définit le nom comme une "entité phonique signifiante» (phônè sèmantikè) ${ }^{13}$, ce qui en fait un signe, mais ce serait un signe particulier, caractérisé par la différence spécifiante d'avoir été établi par convention (kata sunthèkèn). "Symbole» serait précisément le nom de cette différence car, nous dit-il, pour un nom, être établi par convention cela signifie que «rien n'est par nature un nom, mais le devenir c'est devenir un symbole» ${ }^{14}$. Le «sumbolon» est donc, au minimum, un «signe conventionnel», en opposition explicite avec un signe naturel. En tout cas, c'est ainsi que l'ont entendu la plupart de ceux qui tiennent à ce que, dans ce texte, «sumbolon» differe de «sèméion».

Les langues modernes reconnaissent elles aussi au «symbole» le sens d'un «signe conventionnel», dans le contexte des mathématiques ou de la logique par exemple. Cette acception ressemble beaucoup à celle qui paraît ressortir du $D e$ Interpretatione; on a d'ailleurs des raisons de croire qu'elle remonte à une

8. Par exemple le traducteur latin (anonyme ?) de la version parue dans les Operia Omnia (Firmin-Didot, Paris, 1827), ou Cooke (Loeb Classical Library, 1938), qui paraît bien peu sûr de lui, puisqu'il traduit ainsi: "words spoken are symbols or signs of affections or impressions of the soul" - il nous laisse le choix en somme...

9. E.M. Edghill, pour la Oxford Translation of Aristotle, traduit de cette façon en s'appuyant peut-être sur une interprétation analogue à celle d'Aubenque (Le probleme de l'être chez Aristote, $2^{\mathrm{e}}$ éd., Paris, P.U.F., 1977; (1 $1^{\text {re }}$ éd.: 1962), p. 109.

10. Essentiellement celle de Guillaume de Moerbeke, dont la translittération a été reproduite par $\mathrm{G}$. Verbeke dans son édition intégrale de sa version latine du commentaire d'Ammonius (Louvain-Paris, Béatrice-Nauwelaerts, 1961), celles aussi de Tricot (Paris, Vrin, 1959) et d'Ackrill (translation with notes, Oxford, Clarendon Press, 1963).

11. Th. Waitz, Aristotelis Organon Graece, (Hahn, Leipzig, 1844), pp. 324-325.

12. Aubenque, op. cit. p. 108.

13. De Int. 2, 16 à 20-21.

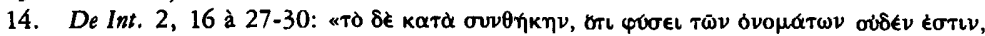

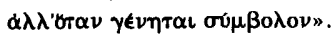


exégèse de ce texte classique ${ }^{15}$; toutefois, une analyse attentive de ces lignes montre également que le «sumbolon» aristotélicien, même s'il rappelle le «symbole/signe conventionnel» moderne, en differe au moins en deux points et que les deux usages ne coïncident pas tout à fait. En premier lieu, on notera que la parole est présentée par Aristote comme un symbole, au même titre que l'écriture, tandis que lorsque nous parlons de "symbole» nous désignons généralement par là un objet visuel et non pas, comme ici, une entité sonore. En second lieu, toujours en suivant la lettre du texte, on remarquera que ce dont l'écriture est présentée comme le symbole, la parole, cela est déjà, par définition, le symbole d'autre chose, le symbole des "pathèmata qui sont dans l'âme». On a donc ici affaire en quelaue sorte à des symboles «au second degré», des symboles de symboles - une façon de s'exprimer qui ne correspond pas non plus à nos habitudes verbales.

Il ne faut pas exagérer l'importance de ces «anomalies». Elles n'en sont d'ailleurs que par rapport à notre propre usage du mot alors que, pour être évaluées, elles devraient être mesurées à l'aune de la langue contemporaine de l'auteur. Voyons donc quel sens possédait «sumbolon» dans le grec antérieur et contemporain d'Aristote.

«Symbole» a commencé par désigner ce qu'on appelle aussi la «tessèré d'hospitalité». C'est un vers de Médée d'Euripide ${ }^{16}$ qui nous informe le plus en détail de cette institution de l'antique hospitalité. Nous y apprenons que, dans les temps archaïques, les hôtes avaient coutume de sceller mutuellement leur alliance en brisant un objet fragile, d'ordinaire un os, dont chacun conservait par devers soi une moitié. Cet objet, considéré dans son unité comme dans ses fragments complémentaires, fut nommé «sumbolon» de «sumballein», «jeter, mettre ensemble». Dans la suite, les «sumbola» servaient à leurs détenteurs pour se reconnaître comme des hôtes. D'abord simples souvenirs, ils étaient également utilisés au bénéfice des descendants des auteurs du pacte. Ils constituaient même une part non négligeable de l'héritage, le patrimoine humain de la lignée en quelque sorte. Grâce à eux, par la simple reconstitution de l'unité originelle, deux hommes parfaitement étrangers l'un pour l'autre, mais liés l'un à l'autre par

15. C'est ce que suggère W. Müri (sú $\mathrm{W} 60 \lambda$ ov. 'Wort-und Sachges-chichtliche Studie. Beilage zum Jahresbericht über das städtische Gymnasium in Bern, 1931), p. 20.

16. Scholia in Euripidem éd. Schwartz, Berlin, Reimer, 1891, vol. II p. 175, 27-32. 
l'amitié ancestrale commémorée par les symboles, pouvaient se saluer comme des hôtes, ce qui n'était pas rien car, pour les Anciens, cette relation privilégiée impliquait une foule d'avantages et d'obligations.

Walter Müri, le seul philologue qui, à ma connaissance, ait étudié cette question par le menu ${ }^{17}$, n'a pas eu de peine à montrer que cette signification originelle commande l'évolution ultérieure du terme. D'abord il prouve que le mot a évolué avec la chose: ainsi, l'extension de l'hospitalité que représente la proxé$n^{n} \mathrm{e}^{18}$, puis le report de la charge qu'elle entraînait des épaules des particuliers aux caisses des Cités-Etats lui permettent d'expliquer le «sumbolon/traité d'État à État» dont nous parlent les écrivains classiques ${ }^{19}$ et le «sumbolon/contrat privé» du grec plus tardif ${ }^{20}$. Puis il rappelle que certains passages difficiles des écrivains attiques, et singulièrement des philosophes ${ }^{21}$, ne peuvent être élucidés que si on renvoie les «sumbola» qu'on y trouve à la «tessera hospitalis» prise comme le paradigme d'un entier qui résulte de la confrontation d'éléments complémentaires. Enfin, c'est toujours de la même source (la tessère étant entendue cette fois-ci comme une preuve d'identité personnelle) qu'il dérive une foule d'usages dont témoignent la littérature et les inscriptions et qu'on peut regrouper sous l'appellation générique «sumbolon/signe probatoire», du «billet de théâtre» à la «preuve d'achat» ${ }^{22}$.

17. Le philosophe américain C.S. Peirce montre lui aussi (voir Collected Papers 2.297) une connaissance surprenante de l'histoire ancienne de ce mot. Le «Greek-English Lexicon» de Liddell and Scott lui a servi de guide.

18. Müri, op. cit., chapitre I.

19. Des orateurs surtout: Démosthène (VII, De L'Halonnèse, II, 9-13) et Andocide (IV, Contre Alcibiade, 18). Sur ces textes et les nuances qu'ils appellent, de même que sur toute cette question, voir Müri, pp. 9-16.

20. Sur le «sumbolon/contrat privé» voir Müri - et en particulier les considérations philologiques des pages 12 à 16 .

21. Parmi les dramaturges, Eubule le Comique dont le scholiaste d'Euripide rapporte un fragment pertinent. Les philosophes auxquels je fais allusion sont Aristote lui-même (Météorologiques II,4 360a 26; De Generatione et Corruptione 331b 37-40, 331b 34, 332a 32; Ethique à Eudeme VII, 5, 1239b 31; De Generatione Animalium I, 18, 722b 11) et Platon, ce dernier pour un seul texte, mais très célèbre, dans le Banquet (191d - 193a). Sur ce texte on consultera les notes de Léon Robin à sa traduction de ce dialogue dans les Belles-Lettres, 1951 ( 5 ème édition, revue et corrigée) pp. 33-34 note 4 et surtout pp. 31-32, note 2.

22. Sur le jeton d'affaire, voir Müri op. cit. pp. 3-5. La métamorphose de la «tessera hospitalis» en «tessera mercantilis» (sic!) a pu s'effectuer très tôt dans l'histoire, comme on peut le conjecturer à partir d'une anecdote que rapporte Hérodote (Erato (VI), 86b). Tous les textes d'Aristote que j'ai cités à la note précédente et qui s'expliquent directement par la «tessera hospitalis» peuvent être aussi rapportés à son «rejeton en ligne directe», le jeton d'affaires, comme le suggère V. Décarie pour Eth. à Eudeme, $1239 \mathrm{~b} 31$, dans sa traduction française (Paris/Montréal, Vrin/Presses de l'Université de Montréal, 1978, p. 172, note 118). 
Aristote a connu et pratiqué la plupart de ces usages. Il est même celui des auteurs classiques qui a contribué le plus à la connaissance que nous en avons: ainsi, quand il nous décrit, dans sa Constitution des Atbéniens ${ }^{23}$, les mécanismes d'utilisation du «symbole de dikaste», une sorte de carte d'identité en forme de jeton qu'on remettait, après leur élection et pour le temps de leur magistrature, à chacun des nombreux juges nécessaires à l'audition d'une cause dans l'Athènes démocratique. Ce jeton était frappé par la cité et il portait une marque conventionnelle.

Où pourrait-on situer le «sumbolon» du De Interpretatione dans les ramifications de cet arbre généalogique? Et d'abord, est-il seul en son genre? Müri soutient l'existence d'une catégorie de «sumbola/signes» dans le grec classique et il la fait dériver directement du verbe «sumballein» qui peut aussi signifier «comprendre» 24 . Je ne pense pas que cela soit nécessaire: la famille des «sumbola» issus de la tessera bospitalis pouvait fort bien engendrer toute seule des synonymes et des quasi-synonymes de «sèméion». C'est particulièrement vrai pour le «sumbolon» qui nous intéresse et dont on sait qu'il est, au moins, un «signe conventionnel». Or, la tessère d'hospitalité et le jeton de dikaste sont empreints d'un caractère conventionnel si marqué qu'il culmine dans la production d'un «sumbolon/contrat», de sorte qu'on doit dire que c'était là juste le mot qu'il fallait pour désigner un signe qui soit en même temps un contrat. Quant à la liste qu'avance Müri, elle témoigne plus contre sa thèse qu'en sa faveur puisque, et il est bien près de le reconnaître lui-même ${ }^{25}$, une bonne part des cas qu'il cite $^{26}$, quand ils ne sont pas indécidables ${ }^{27}$, peuvent être facilement rattachés à l'une ou l'autre des branches de l'arbre qu'il a décrit dans les pages précédentes. Quoi qu'il en soit de l'origine particulière de chacun des éléments de cet enisemble, chaque cas mérite donc d'être examiné pour lui-même, à part des autres.

23. Constitution des Atbéniens LXV 2; LXVIII 2; LXIX 2.

24. Comme dans l'expression $« \sigma v \mu \beta \alpha \lambda \in i \nu \mu \alpha \nu i \in i \alpha \nu »$ (interpréter un oracle). Voir Cratyle, ! 384 a.

25. P. 18: "Oft zeigt sich (...) der Einfluss der in der Sprache gleichzeitig lebendige Homonyme. Manchmal zeichnet er sich als schwache Färbung ab; doch auch wieder so, dass man eine eigentliche Verschmelzung der Vorstellungen behaupten kann."

26. Müri, pp. 18 et ss.

27. C'est le cas, à mon sens, du vers 1150 de Théognis, dont on peut proposer plusieurs lectures mais dont aucune n'est décisive, le fragment orphique no. 23 de la numérotation de Diels-Kranz, qui est trop endommagé pour qu'on puisse en tirer quelque chose etc. 
En ce qui concerne le «sumbolon» qui m'intéresse, celui du De Interpretatione, je pourrais m'arrêter là. Cependant j'ai des raisons de me méfier de l'interprétation qui réduit ce «symbole» à un «signe conventionnel». D'abord, il ne faut pas perdre de vue qu'en lisant «sumbolon» de cette façon on utilise un concept qu'on a extrait en large part du texte même qu'il s'agit d'éclairer. Qui plus est, en faisant cela, on va à contre-courant de ce qui paraît avoir été la démarche de l'auteur, qui n'a pas cherché à expliquer le symbole par la convention mais, tout au contraire, à clarifier la convention par le symbole. En effet, quand Aristote parle de symbole pour la seconde fois c'est à l'occasion d'un retour sur le conventionnalisme qu'il a introduit comme un des éléments de sa définition du nom, un retour qui est bien une sorte de clarification de ce point de la définition. Or, si l'on s'en tient à la lettre du texte, «symbole» serait précisément le pivot de cette explication: si on peut dire des noms qu'ils ont été établis par convention, c'est "parce que (oti) rien n'est par nature un nom, mais le devient quand il devient un symbole» ${ }^{28}$. On peut s'étonner de ce supplément d'information. Ayant en main le Cratyle de Platon, nous regardons l'idée que les noms ont été établis par convention comme une idée familière, un thème courant de la discussion philosophique en Grèce aux Vième et IVième siècle. S'il faut limiter notre compréhension de ce «symbole» à celle d'une convention de sens, on doit s'interroger sur la valeur de l'explication qui se construit autour de ce concept: dire qu' «être établi par convention, pour un nom, cela signifie que rien n'en est un par nature, mais le devient quand il fait l'objet d'une convention ou d'un contrat» ce n'est pas dire grand-chose.

Je préfere voir dans ce symbole une contribution plus substantielle. Je suppose qu'avec cette mention du symbole Aristote voulait ajouter une touche nouvelle et significative à une thèse bien connue, qu'une explication détaillée de ce concept devait être fournie dans son enseignement, un enseignement dont les cinq premiers chapitres du De Interpretatione - textes très schématiques qui en suggèrent plus qu'ils n'en disent - constitueraient la charpente (au sens où le syllabus d'un cours en constitue la charpente) et que cette doctrine, même si son exposé n'a pas été conservé dans les textes, doit au moins y avoir laissé des traces.

28. Voir note 18. 
En dehors du De Interpretatione, «sumbolon», dans le sens qui m'intéresse, revient deux fois dans toute l'oeuvre d'Aristote.

Du premier de ces textes, tiré du premier chapitre des Réfutations Sophistiques, nous pouvons extraire la différence essentielle des choses et des symboles. Le De Interpretatione nous a déjà appris qu'entre les choses et la pensée que nous avons de ces choses il y a ressemblance, ce qui signifie que tous les locuteurs vivent dans le même monide et le conçoivent tous à peu près de la même façon. Par contre, pour ce qui est du rapport des mots et des choses, les Réfutations Sopbistiques précisent qu'entre eux «il n'y a pas de ressemblance complète: les noms sont en nombre limité, ainsi que la pluralité des définitions, tandis que les choses sont infinies en nombre. Il est, par suite, inévitable que plusieurs choses soient signifiées (sèmainein) et par une même définition et par un seul et même nom ${ }^{29}$.» Cette disparité des choses et des mots résulte, nous dit Aristote, de la nature même du langage. En effet, si nous devons faire usage de la parole «c'est parce qu'il n'est pas possible d'apporter dans la discussion les choses ellesmêmes, mais qu'au lieu des choses nous devons nous servir de leurs noms comme de symboles» ${ }^{30}$.

L'être se présente donc.autrement dans le langage que dans le monde des sens et le symbole, étant opposé à la chose, se retrouve au coeur de cette altérité. Les "choses» sont toujours singulières, d'un certain temps et d'un certain espace, tandis que ce vers quoi pointent les symboles est universel, n'est attaché à aucun temps et à aucun espace et ne correspond pas à des choses empiriques mais à des définitions. La communauté de définition rassemble sous un même nom une infinité de choses diverses mais semblables pour «l'essentiel» et le symbole est proprement le foyer unique sous lequel on peut subsumer le multiple des étants qui partagent «l'essentiel», qui répondent à la même définition. Les noms-symboles ne se rapportent donc pas aux choses ellesmêmes mais à la pensée que nous en concevons, plus précisément

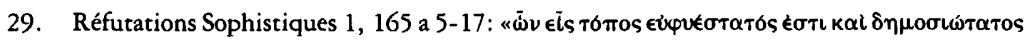

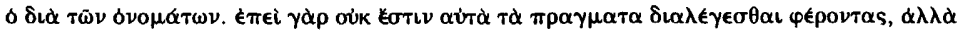

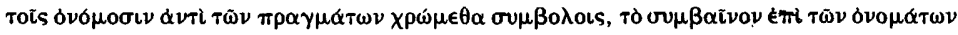

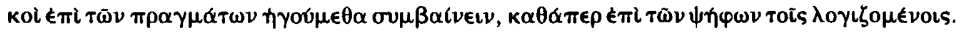

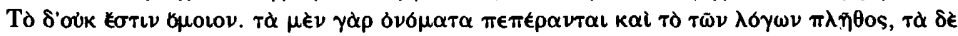

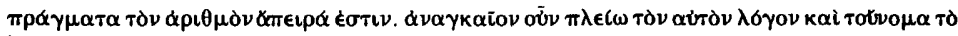
È on

30. Réfutations Sophistiques 1,165 a 6-8. 
à ce type de pensée des choses qu'est leur pensée sur un mode universel, nous dirions aujourd'hui leur concept.

Pour communiquer, nous devons passer nécessairement par l'intermédiaire du concept mais nous ne communiquons pas seulement des concepts. De même que la substance peut être située, qualifiée, temporalisée par ses accidents, de même le concept peut parvenir à faire porter la pensée sur un individu par l'adjonction au symbole d'autres "phônai» et en particulier du verbe $^{31}$.

J'ai dit au début qu'Aristote donnait une saveur biologique à sa définition du langage en l'y présentant encore encastré dans son support sensible, la voix. Le second texte pertinent à ma recherche et extérieur au De Interpretatione, qui est tiré du De Sensu (un traité de biologie précisément), me permettra de faire ressortir l'importance de cette remarque et, enfin, de tenir ma promesse, faire entrer l'étude du langage dans les recherches anthropologiques et biologiques d'Aristote par le moyen du «sumbolon».

Dans le De Sensu ${ }^{32}$, donc, nous lisons que «par rapport à la vue, l'ouie n'est la cause de l'acquisition d'un plus grand nombre de connaissances qu'en vertu du langage. Or le langage est une composition de mots (onomata) et les mots eux-mêmes ne sont que des symboles.» La primauté accidentelle de l'ouie provient donc de ce que toute l'expérience possible, intellectuelle ou sensible, peut être dite, mise en langage, traduite en paroles, réduite à des compositions d'éléments sonores. Le point de vue de l'auteur du De Sensu étant celui d'un biologiste, pour tirer le maximum de cette dernière occurrence, il faut l'interpréter en référence étroite avec ce qu'il dit un peu auparavant de la nature et de l'utilité des sens de l'ouie et de la vue. Ces sensations appartiennent aux animaux doués de locomotion et elles leur servent à s'orienter dans leur action, «elles sont pour eux un moyen de préservation, de sorte que guidés par une sensation précédente ils peuvent se mettre à la poursuite de leur proie et aussi éviter ce qui est inférieur ou pourrait les détruire ${ }^{33}$.» Cette propriété leur vient

\footnotetext{
31. La définition du nom, je le rospitalappelle, stipule qu'il ne comporte aucune sorte de

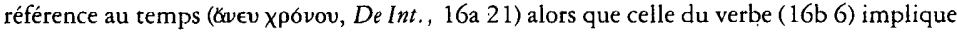
essentiellement cette référence (

32. De Sensu, 1, 437 a 11-15.

33. De Sensu, 1, 436 b 16 et ss.
} 
du fait qu'elles sont des sensations qui opèrent à distance de leur objet et non pas, comme le toucher et le goût, des modes de perception qui nécessitent un contact physique immédiat du sens et du sensible.

Chez l'homme, cette faculté de se construire une configuration plus large de son environnement grâce à l'ouie et à la vue atteint son utilité maximale puisque le langage lui permet de tout traduire en sons et subséquemment en écriture. L'ouie et la vue, l'ouie tout spécialement, seront les sens pour lesquels il y aura des symboles. Par la parole, je puis, en un sens, vous faire toucher, voir, entendre, sentir, goûter des choses qu'il serait impossible, difficle, peu pratique ou dangereux de percevoir directement, par exemple le temps qu'il fait à la surface de Vénus ou la saveur un peu trop puissante de ce condiment dont vous alliez témérairement ingurgiter une trop généreuse portion et contre lequel je vous mets en garde. 'Il semble qu'il n'y a presque rien qu'on ne puisse dire, dont on ne puisse parler: de la pluie qu'on a eue hier, de celle qu'on aura peut-être demain, de celle qui tombe sans doute à l'heure actuelle en Patagonie, de celle qui tomberait parfois sur le pays des Fées, si un tel pays existait etc . . . Grâce à l'ouie, alors que ce seul sens est engagé, nous pouvons vivre l'expérience «seconde», «l'expérience psychique» de sons (qui ne sont pas ceux que nous entendons), d'odeurs, de saveurs, de ductilités, de visions de choses ou d'événements qui tombent en dehors de notre expérience actuelle, mais qui sont autant d'informations utiles ou même indispensables à la poursuite et à la planification de notre existence.

Le De Sensu appartient à la collection des traités de sciences naturelles. Aristote avait sans doute plusieurs raisons de s'intéresser aux êtres vivants, mais l'une des plus marquantes de ses préoccupations, qui se fait jour dans le De Partibus Animalium; l'Historia Animalium, le De Generatione Animalium, le De Anima, le De Memoria et le De Sensu (donc les plus importants textes de cette collection), était de situer l'homme dans le règne animal. Or, on voyait cette préoccupation se manifester déjà dans le second chapitre du De Interpretatione et précisément à propos du symbole. 
En effet, dans le contexte de l'étude du nom, le «symbole» se situe au foyer d'une double opposition, entre la nature et la convention d'une part, l'animalité et l'humanité d'autre part: après avoir dit que «rien n'est par nature un nom mais le devient quand il devient un symbole», Aristote ajoute, comme pour justifier cette affirmation, qu'il n'en est pas ainsi «des sons inarticulés comme ceux qu'émettent les bêtes» car ceux-ci «même s'ils manifestent (dèlousi) quelque chose ne sont pas pour autant des noms ${ }^{34}$.»

Il semble donc que la précision du concept de symbole passe par une comparaison de l'humanité et de l'animalité sur un plan biologique. De fait, si Aristote emploie «sèméion» dans une grande variété de contextes, il réserve «sumbolon» exclusivement au langage humain. Il devient alors capital de pouvoir distinguer ce qui fait la capacité de créer des symboles, de parler un langage, du simple pouvoir d'émettre des signes ou des cris, s'il est vrai que, face aux autres vivants, l'homme se définit essentiellement comme un «zôon logikon», un "animal logique»" 35 , ce qui peut autant vouloir dire «animal parlant» qu' «animal raisonnable». Raison et langage ne se confondent pas ici en un seul mot par hasard: les deux facultés distinctes dont le développement fait l'excellence de l'homme, la puissance de théoriser (la raison théorique) et le pouvoir de délibérer prudemment (la raison pratique), correspondent à deux usages du langage. Le De Sensu nous le rappelle $e^{36}$ et ce que nous avons dit du symbole nous permet de le comprendre. L'énoncé théorique type conserve la modalité universelle du symbole autour duquel il se construit. L'énoncé délibératif, par contre, inscrit le concept dans une visée spatio-temporelle particulière et définie. Il va donc de soi que si l'homme est le seul de tous les animaux à pouvoir parler c'est d'abord parce qu'il est le seul à pouvoir théoriser ou délibérer. Mais comment apprend-il à parler en tout premier lieu? Il est probable qu'Aristote n'a jamais tenté de résoudre cette question, mais il nous a cependant donné les moyens de penser la faculté à partir de laquelle l'homme peut parler.

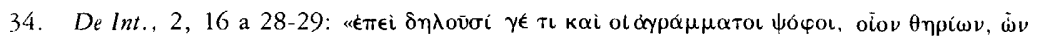

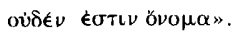

35. L'expression se trouve dans un fragment (no. 187 de la classification de Rose).

36. De Sensu, 1, 437 a 1-4. 
Le De Memoria nous dit que l'homme possède deux mémoires, la mnémie ${ }^{37}$, qu'il partage avec les autres animaux supérieurs, et l'anamnèse ${ }^{38}$, qui lui est propre. Par la mnémie, nous nous souvenons en réponse à un stimulus externe. Par anamnèse, nous nous remémorons volontairement, nous sommes la propre cause de nos pensées. Il est évident que nous nous faisons entendre des autres en manipulant leur mémoire (mnémie): notre voix agit sur leur âme à la manière d'un stimulus qui suscite en elle les images ou représentations auxquelles correspondent conventionnellement les symboles audibles qu'elle profere. Il est aussi clair que c'est l'anamnèse qui fonde notre pouvoir de nous souvenir des mots mêmes que nous devons utiliser. "Chercher ses mots", c'est essayer de se les rappeler. Ainsi donc l'anamnèse, mémoire volontaire, n'est pas seulement le pouvoir d'établir des conventions de sens: elle est la condition indispensable de la conservation ultérieure des conventions établies à un moment donné.

Cette dernière différence entre l'homme et l'animal nous fait enfin comprendre pourquoi Aristote a sélectionné le mot «symbole» pour désigner l'élément fondateur du langage: la tessère d'hospitalité était cet artifice de commémoration par lequel, à travers le temps, nous maintenons un lien originel et autour duquel se construit une amitié; de même le symbole sera aussi un outil conventionnel de souvenir grâce auquel se fixent et se renforcent les points de repère conceptuels qui déterminent notre rapport au monde et même constituent pour nous ce monde d'essences stables, un monde où il y a une humanité. Pour nommer cela, il n'y avait pas de meilleur terme à métaphoriser que celui qui désignait le plus beau des pactes qui unissent les hommes et qui sert à préserver leur union par-delà la fuite du temps.

J'ai évoqué un peu plus haut la structure complexe de la proposition en suggérant que le nom, ou au moins un des noms contenus dans la proposition, y joue un rôle analogue àcelui de la substance, substratum en lui-même non temporel mais dont on prédique le changement, que ses accidents temporalisent. Le rapprochement s'impose même dans la mesure où le langage est

37. De Memoria, I, ( $\mu \nu \eta \mu \eta)$.

38. De Memoria, II, ( $\alpha \nu \alpha \mu \nu \eta \sigma \iota s)$, et en particulier 453 a 5-14. 
symbole fidèle de ce qui est pensé dans l'âme. Or, l'âme peut penser l'extériorité comme un monde de substances stables. Cela lui est même indispensable, apparemment, si elle veut se représenter ce que c'est que le temps et l'ordonner en passé et futur de part et d'autre d'un «maintenant» toujours «autre et autre». On sait en effet que la représentation adéquate du temps dépend de la représentation d'un mouvement et qu'un mouvement, pour avoir de l'unité, doit être envisagé comme le mouvement dont est affecté une même substance, "sujet du mouvement»" 39 . Là encore, il y a isomorphie entre la structure de la proposition telle qu'elle est présentée dans le De Interpretatione, une composition comportant au minimum un nom «sans référence au temps» et un verbe temporalisant cette substance, et la structure de la représentation du mouvement, où il y a également un élément fixe par rapport auquel on se représente le changement. Ce n'est pas par hasard que, dans l'un et l'autre cas, on parle de «sujets».

De là, on est amené naturellement à chercher dans l'âme le principe commun de ces deux structurations isomorphes, le pouvoir de parler et le pouvoir de se représenter le temps, tous deux propres à l'homme. Ce principe ou cette faculté pourrait bien être cette sorte de mémoire dont j'ai parlé (et l'imagination qui lui est étroitement liée) qui nous permet de nous abstraire par la pensée des sollicitations immédiates du présent et de déterminer notre action en recourant à la médiation d'images se rapportant soit au passé, soit à l'avenir, soit à un état permanent ou persistant des choses.

Toutefois, je doute qu'on puisse s'en tirer à si bon compte. Il y a en effet, dans la pensée, une différence entre la pensée du divers et la pensée du même. Le langage n'est pas symbole de n'importe quelle sorte de pensée et même lorsqu'il renvoie à du divers singulier, il ne peut faire l'économie de l'intermédiaire du même puisque c'est une définition qui transcende sa singularité qui fait qu'on peut donner tel nom à tel singulier. Bien plus, on peut se demander si une pensée constituée peut même avoir accès à quelque chose comme du "pur divers», une diversité qui ne serait en rien affectée par ce dont le langage est symbole. La question qui se pose est celle de l'origine du concept: qu'est-ce qui

39. «Le mû», Physique, IV, 11. 
entre en acte en premier lieu, la pensée ou le langage? Est-on d'abord en possession de la pensée du même avant de la symboliser dans le discours ou, au contraire, est-ce en apprenant à parler qu'on commence à penser le monde?

L'entreprise de répondre à cela à partir d'Aristote nous mènerait loin dans les ramifications de sa pensée. Quant à s'attaquer à la question pour elle-même, cela exigerait de celui qui s'en chargerait qu'il fournisse l'effort de constituer une philosophie du langage qui assumerait entièrement le locuteur, qui serait même centrée sur lui.

Département de philosophie

Université de Montréal 